\title{
Gerontological Nurse Practitioner
}

National Cancer Institute

\section{Source}

National Cancer Institute. Gerontological Nurse Practitioner. NCI Thesaurus. Code C71291.

A registered nurse with specialization and higher level of training in treating the elderly population. This includes the management of acute and chronic illness, as well as addressing the psychosocial issues that can be present with aging. Gerontological Nurse Practitioners are employed in clinics, doctor's offices, long-term care facilities, and hospitals. 